\title{
An Assessment of the Solar Potential of Roofs within a Web-based Solar Cadastre
}

\author{
Ekaterina Chuprikova ${ }^{1}$, Armin $\operatorname{Costa}^{1}$, Markus Innerebner ${ }^{1}$, Roberto Monsorno ${ }^{1}$, \\ Jochen Ernst Wagner ${ }^{1}$, David Moser ${ }^{2}$ and Mark Zebish ${ }^{1}$ \\ ${ }^{1}$ EURAC, Institute for Applied Remote Sensing, Bozen/Italy·ekaterina.chuprikova@eurac.edu \\ ${ }^{2}$ EURAC, Institute for Renewable Energy, Bozen/Italy
}

Short paper

\begin{abstract}
Solar technologies offer clean and sustainable energy production at an affordable cost. Therefore, their use is rapidly growing, and private users as well as local authorities demonstrate wide interest in identifying areas suitable for mounting solar modules. The recent improvements of remote sensing techniques such as LIDAR (Light Detection and Ranging), are very attractive and suitable for solar potential assessment; however, it is of paramount importance to transfer the knowledge to the public. The proposed solution includes the development of an interactive tool, Solar WebGIS, which can analyse and visualize solar potential characteristics. Furthermore, the article discusses the data preparation, integration, and visualization issue related to the WebGIS.
\end{abstract}

\section{Introduction}

In the last decade, the energy issue has become a crucial component of ecologically and economically sustainable urban development. The conventional energy sources (e.g. fossil fuel based) are tending to decrease, while renewable energy sources are making an increasing contribution. Although solar technologies are quite mature already, and photovoltaic and thermal solar systems are available for private users and have gained high importance for industrial use, there is a lack of information for the preliminary estimation of solar power. The estimation of the PV potential at roof level is of particular interest, due to the trend of PV installations in Italy, where the market has been moved from open field plants towards building integration (MOSER et al. 2014).

The proposed solution includes the development of the Solar WebGIS application, a stateof-the-art technology, which offers immediate access to the information about solar radiation distribution on building roofs within a single intuitive Web environment. To proceed with this task, the information should be merged and visualized in a convenient way for the end user, which is not trivial. The initial data, the digital terrain model (DTM) and digital surface model (DSM), are derived from the LIDAR data, and provided as input information for solar radiation simulations. The approach incorporates the workflow for convenient and successful analysis of large amounts of spatial data, including the integration of different formats such as vector and raster, and it allows query operations and spatial analysis. The Solar WebGIS has been developed within an existing Spatial Data Infrastructure (SDI) that provides easy access for collaboration and distribution of solar potential data. 
The research is applied to South Tyrol, an Alpine region in Northern Italy. The investigated area has great potential for developing solar energy due to a favourable geographic location, and policy measures designed to improve the energy efficiency. The combination of cool temperatures and large amounts of solar radiation provides ideal conditions for the solar power systems. However, the complex Alpine landscapes make an assessment of solar potential challenging. The objective of the application is to increase environmental awareness among the residents and provide private users and authorities with comprehensive information for the planning, installation, and investments in the solar power systems within a single intuitive Web environment.

\section{WebGIS Implementation}

The solution includes work on the combination of vector and raster data, which allows the incoming solar radiation hitting the rooftops and shading to be evaluated at any given location. The initial data consists of precise surface and terrain models, namely the DTM and DSM with a resolution of 5 meters, derived from the LIDAR data available for the main valleys. This data served as the input for evaluating the amount of solar irradiance that falls on each unit of surface every 15 minutes, and is used for determining its individual potential for solar electric generation using photovoltaic (PV) panels.

The DTM and DSM were reprocessed using the r.sun module within the open-source software GRASS GIS, for the estimation of solar irradiance. The solar irradiance, incident to the Earth's surface, is a result of complex interactions of the light between the atmosphere and surface (Š́́RI \& HOFIERKA 2004). Therefore, the main factors influencing the solar irradiance are the position of the Earth with respect to the sun, atmospheric impacts, and terrain surface. The r.sun module allows for these three factors to be accounted for. The impact of clouds is taken into account by multiplication with the average daily cloud index for the period 2004 to 2013 (value between 0 and 1). The processing was performed according to a tiling system that was introduced in order to support the estimation based on bigger tiles, and to account for shadowing. Eventually, the yearly irradiation $(\mathrm{Wh} / \mathrm{m} 2)$ was derived based on daily global solar irradiation. Since the efficiency of photovoltaic modules highly depends on temperature, it is necessary to estimate the average temperature within the area of interest. According to the method described by HuLD et al. 2006, the average temperature diurnal cycle can be approximated as a curve (or a set of points) representing the typical variation of the temperature within a day for a given location. An estimate of the average temperature during the daytime can be derived by integrating daily temperature values between sunrise and sunset. Additionally, main data sources are cadastre data and vector data of protected areas. The estimation of photovoltaic characteristics is based on the algorithm developed by HULD et al. 2010, and experimental work as conducted in the SoLaRE-PV laboratory of the Institute for Renewable Energy, EURAC. The algorithm allows working with different PV technologies, and it takes into account several variables, in particular temperature of the module, ambient temperature, and irradiance, giving electrical power $P_{P V}\left[\mathrm{~W} / \mathrm{m}^{2}\right]$ of $\mathrm{PV}$ modules as the output value.

The implementation of the storage for the large amounts of data is particularly challenging in the following research. All data is organized in a spatial database, hence it can be manipulated, retrieved, and related to all other stored data. The irradiance and temperature 
information are incorporated in raster format, and are retrieved via spatial query. The database stores the data in a byte column, with a reference to a separate raster table.

Apart from the estimation of the solar potential, it is of paramount importance to transfer the knowledge to the public. There are plenty of tools that can represent the data; however, WebGIS can provide a convenient and comprehensive way to communicate the information, and offer access to the information integrating different data sources. Therefore, a wide audience of users can retrieve the details about an object in the single user interface, with no need for additional software installation. Besides, based on the open-source software and application programming interfaces (APIs), there is a big potential to create rich and interactive applications.

The WebGIS application has a three-tier architecture. The front-end (presentation layer) is incorporated in a Web browser, and the implementation of the layout and interaction is done in JavaScript, JSP, and CSS. The middleware (application layer) component uses Apache as the Web server and Apache Tomcat as the Servlet Engine. On the Servlet Engine, two servlet containers are present: the rendering engine runs on one container (GeoServer), and the other container is responsible for the configurations of the WebGIS. The configurations are stored in a XML configuration file, and include client and server side settings. The back-end (data layer) comprises the access to file data sets (raster or vector), and to a DBMS. The entire WebGIS architecture is completely based on Open Source Software without any license restriction.
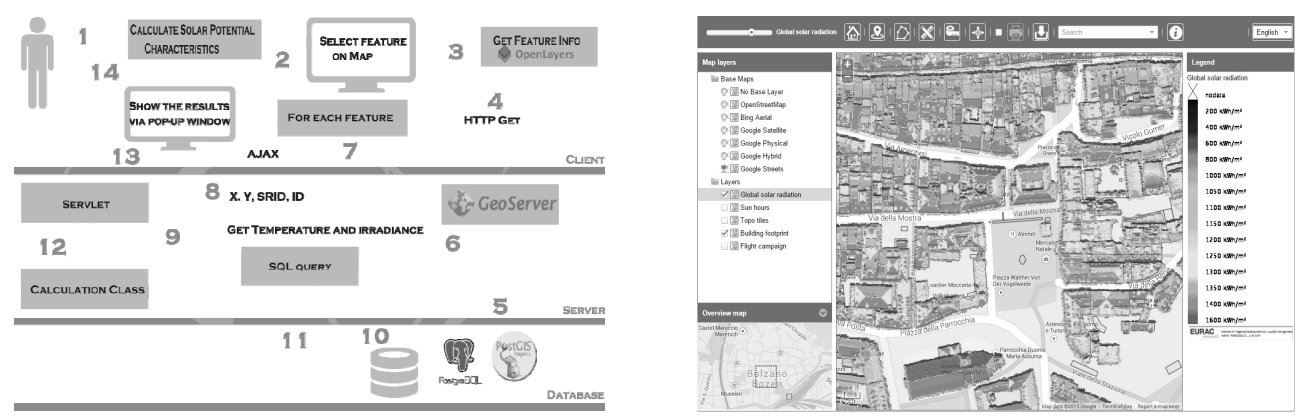

Fig. 1: Solar WebGIS: Solar potential estimation within the application (left) and interface of Solar WebGIS (right)

The Solar WebGIS offers different options to the user through various features, as for example overlay with thematic layers, measuring tool, printing option, data download, identification tool, and metadata overview, retrieved from a spatial catalogue. The server and client architectures for Solar Cadastre are designed in a close connection. The server primarily retrieves information from the database by means of a query, produces estimations of the solar potential, generates a response, and sends it back to the client.

The workflow of the application is presented in Figure 1. To estimate solar potential characteristics, a user selects an object of interest. The "get feature" request returns a selection of features from the data source. For each feature, the parameters (coordinates, SRID, ID) are sent to the server. The Servlet triggers an SQL query that requests temperature and irradiance values from the database according to the parsed parameters, and returns corre- 
sponding values from the raster table. Therefore, the Servlet contains such parameters as irradiance and temperature. The constant values are the number of days and hours, module size, temperature of module cell, and parameters of different PV technologies. The Calculation Class contains equations for estimation of efficiency, electricity, emissions savings, and energy production. Hence, the estimations are performed in the Calculation Class based on the parsed parameters. The final results are parsed to the Servlet, which creates a JSON object and sends it to the client. Eventually, the results are visualized in the client component (Web browser) via pop-up window.

\section{Results}

The following research has been conducted to support the decision-making process for the evaluation of the suitability of solar panel installation. The Solar WebGIS demonstrates the use of modern Web standards and development practices to create an interactive user interface for Web-based environmental modelling. For practical applications, Solar Cadastre can be used as a fast visualization for investigating the solar potential of rooftops. The Webbased application provides a quick and easy way to evaluate a solar power system, before turning to thorough investigations provided by installing and planning companies. Moreover, one of the concerns of this research is to develop an approach that allows storage of large amounts of data, and performs various estimations for photovoltaic assessment. The Solar WebGIS is a client/server application that was developed using free and open-source software libraries, frameworks, and current Web standards. When it comes to future development, it is of paramount importance to optimize the data, and introduce new characteristics such as solar hours, slope, and aspect, which can enhance the informative content of the application, and improve the estimations. Besides the initial installation costs, monthly revenue and available installers could be valuable information for many users. Based on this data, the decision for further work towards solar energy implementations can be made. Eventually, support of mobile devices would be an issue of interest to improve field operations, and to make better-informed business decisions.

Acknowledgement: The SolarTirol project is co-financed by the European Regional Development Fund (ERDF) (Interreg IV 2007-2013 Italy-Austria).

\section{References}

Huld, T. A., Gottschalg, R., Beyer, H. G. \& Topič, M. (2010), Mapping the performance of PV modules, effects of module type and data averaging, Solar Engy., 84 (2), 324-338.

Huld, T. A., Šúri, M., DunloP, E. D. \& MmiCALE, F. (2006), Estimating average daytime and daily temperature profiles within Europe. Environmental Modelling and Software, 21, 1650-1661.

Moser, D., Vettorato, D., Vaccaro, R., Del Buono, M. \& Sparber, W. (2014), The PV Potential of South Tyrol: An Intelligent Use of Space. Energy Procedia, 57, 1392-1400.

ŠÚRI, M. \& HOFIERKA, J. (2004), A new GIS-based solar radiation model and its application to photovoltaic assessments. Transactions in GIS, 8 (2), 175-190. 